\title{
La politique de la santé part en vacances
}

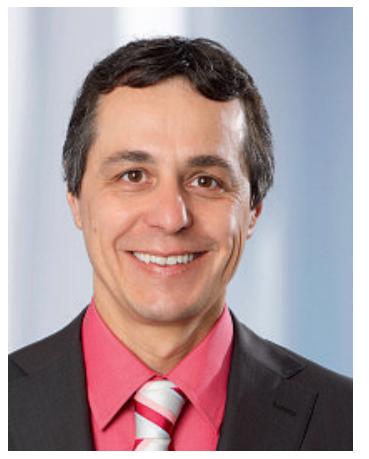

Il n'y a pas que la politique qui réchauffe le climat! Les derniers jours nous ont annoncé l'été et nous nous réjouissons tous d'un peu de détente estivale, avec ses musiques, ses parfums, ses soirées sur une terrasse au bord de l'eau et ses fruits de mer. La politique part en vacances, les politiciens remplissent leurs poumons d'air et se préparent pour le rush électoral qui débutera en août. Ceux qui quittent le Parlement vont profiter de cette pause pour vider leurs tiroirs et s'imaginer un avenir. Ceux qui trépignent à l'idée de conquérir le Palais vont préparer les détails de leur campagne électorale. En septembre, l'automne amènera une émotion nostalgique sur la session; pour certains ce sera la session des adieux et pour d'autres celle des au revoir, avec son lot d'incertitudes, car personne n'est sûr de rien en politique.

Les partants seront heureux de quitter la scène avant le lamento habituel des primes de caisse-maladie. Les candidats investiront les spin doctors, les interviews plus ou moins amicaux, les budgets de campagne (fatalement insuffisants) et la surveillance des concurrents. La hausse des primes ne pourra les retenir que dans l'optique de leur campagne électorale. En cas contraire, «Circulez: il n'y a rien à voir!»: on y pensera demain! C'est ainsi: «the show must go on» et, entre une polémique et l'autre, le 23 octobre arrivera vite et nous dévoilera la nouvelle vision du monde que portera notre Assemblée fédérale.

\section{La décision sur le nouveau système de contrôle de l'économicité des prestations médicales a été repoussée à la session de septembre.}

Entretemps, plus personne ne se souviendra de la session d'été qui s'est achevée ce 17 juin, ni des décisions qui y ont été prises (ou pas). La Chambre des cantons a lutté contre les cantons eux-mêmes, du jamais vu, pour modifier à minuit moins une la réforme de la LAMal sur le financement hospitalier. Non pas au sujet des swissDRG, mais de la répartition du financement des hôpitaux entre impôts et primes. Le Conseil national a ensuite tranché en faveur des cantons: le monde à l'envers!
La décision que le Parlement n'a malheureusement pas prise concerne le nouveau système de contrôle de l'économicité des prestations médicales: on l'attendait avec impatience, mais une trop longue discussion sur la nouvelle loi de coordination des hautes écoles universitaires a repoussé ce dossier à septembre prochain.

La Commission de la sécurité sociale et de la santé publique du Conseil national, dans sa réunion du 23 et 24 juin, a examiné en dernière lecture la révision de la LAMal sur les

\section{Soins intégrés: lors de la prochaine session, il s'agira de trouver un accord sur la participation aux coûts, l'indépendance des médecins et l'obligation des assureurs d'offrir du Managed care.}

soins intégrés: cette révision trouvera ainsi son épilogue le 30 septembre prochain. La conclusion pourra être heureuse - donc la loi acceptée - à condition de trouver un accord sur les deux divergences et demie qui demeurent entre les Chambres: la participation aux coûts, l'indépendance des médecins et l'obligation des assureurs d'offrir du Managed care (ce dernier point est une demi-divergence, puisqu'il est déjà pris en compte dans les normes transitoires). Je suis plutôt optimiste sur l'évolution de ce projet, peut-être à cause de l'été qui nous attend, ou car j'ai envie de dire comme Winston Churchill: «Un pessimiste voit la difficulté dans chaque opportunité, un optimiste voit l'opportunité dans chaque difficulté.» Même au-delà du cap du Parlement, tout ne sera pas terminé: par un référendum, c'est la population suisse qui tranchera définitivement sur cette importante innovation. Et le nouveau Parlement recommencera, avec l'enthousiasme des regards neufs, à étudier toutes sortes de solutions, afin de mettre en place le meilleur équilibre possible entre nos attentes démesurées face au système de soins et notre disponibilité modeste à le financer.

Rien de ceci n'est nouveau, et pour l'instant il y a une autre priorité, c'est la promotion de la santé: la plage nous attend, allons-y!

Dr Ignazio Cassis, vice-président de la FMH et conseiller national 